Neurosurg Focus 26 (2):E8, 2009

\title{
Phototherapy in peripheral nerve regeneration: From basic science to clinical study
}

\author{
SHIMON RochKind, M.D. \\ Division of Peripheral Nerve Reconstruction, Department of Neurosurgery, \\ Tel Aviv Sourasky Medical Center, Tel Aviv University, Tel Aviv, Israel
}

\begin{abstract}
Object. This review summarizes the continuous study of low-power laser radiation treatment of a severely injured peripheral nerve. Laser phototherapy was applied as a supportive factor for accelerating and enhancing axonal growth and regeneration after injury or a reconstructive peripheral nerve procedure. In nerve cell cultures, laser phototherapy was used to stimulate activation of nerve cells.

Methods. Low-power laser radiation was used for treatment of peripheral nerve injury using a rat sciatic nerve model after crush injury, neurorrhaphy, or neurotube reconstruction. Nerve cell growth and axonal sprouting were investigated using laser phototherapy on embryonic rat brain cultures. The outcome in animal studies facilitated a clinical double-blind, placebo-controlled, randomized study that measured the effectiveness of 780-nm laser phototherapy on patients suffering from incomplete peripheral nerve injuries for 6 months to several years.

Results. Animal studies showed that laser phototherapy has an immediate protective effect, maintains functional activity of the injured nerve, decreases scar tissue formation at the injury site, decreases degeneration in corresponding motor neurons of the spinal cord, and significantly increases axonal growth and myelinization. In cell cultures, laser irradiation accelerates migration, nerve cell growth, and fiber sprouting. A pilot clinical double-blind, placebocontrolled, randomized study showed that in patients with incomplete long-term peripheral nerve injury, 780-nm laser radiation can progressively improve peripheral nerve function, which leads to significant functional recovery.

Conclusions. Using 780-nm laser phototherapy accelerates and enhances axonal growth and regeneration after injury or a reconstructive peripheral nerve procedure. Laser activation of nerve cells, their growth, and axonal sprouting can be considered as potential treatment of neuronal injury. Animal and clinical studies show the promoting action of phototherapy on peripheral nerve regeneration, making it possible to suggest that the time for broader clinical trials has arrived. (DOI: 10.3171/FOC.2009.26.2.E8)
\end{abstract}

KEY WoRDS $\bullet$ axonal sprouting $\bullet \quad$ laser phototherapy $\quad \bullet \quad$ nerve cells
peripheral nerve injury $\quad$ rat

$\mathrm{I}$ NJURY of a peripheral nerve frequently results in considerable disability. In an extremity, such lesions may be associated with loss of sensory and motor functions, which leads to severe occupational and social consequences. Surgical repair is the preferred modality of treatment for the complete or severe peripheral nerve injury. ${ }^{2,8,9,11,27-29}$ In most cases, the repair can be successful if the surgery is performed in the first 6 months after injury, in comparison with long-term cases in which surgical treatment is less successful. Nonetheless, in related literature, there are several publications involving surgical treatment of long-term injuries of the brachial plexus and peripheral nerve. ${ }^{7,10,13,18}$ For most patients who suffered from long-term peripheral nerve injuries, the continuation of rehabilitation therapy was recommended, especially in those regions or countries that don't have specially dedicated peripheral nerve surgeons. Unfortunately, spontaneous recovery of long-term, severe, incomplete peripheral nerve injury is often unsatisfactory.
The usual results after such an injury are degeneration of the axons and retrograde degeneration of the corresponding neurons of the spinal cord, followed by a very slow regeneration. Recovery may eventually occur, but it is slow and frequently incomplete. Understandably, therefore, numerous attempts have been made to enhance and/ or accelerate the recovery of injured peripheral nerves.

Among the various proposed methods for enhancing nerve repair, phototherapy has received increasing attention over the past 2 decades. The term phototherapy refers to the use of light for producing a therapeutic effect on living tissues. Although a pioneering report of the effects of laser phototherapy on the regeneration of traumatically injured peripheral nerves was published in the late $1970 s,{ }^{12}$ it is only since the late 1980 s that scientific interest was kindled for this therapeutic approach to neural rehabilitation, leading to the publication of a number of studies that have shown positive effects of phototherapy on peripheral nerve regeneration. ${ }^{6}$ 

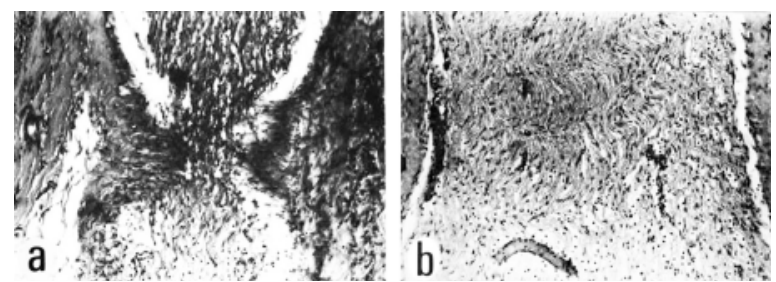

FIG. 1. Histological section of the crushed area of a rat sciatic nerve showing the response of the nerve to laser phototherapy. a: Nonirradiated nerve. Note the scar ring of fibrous tissue. b: Lasertreated nerve shows no visible scar. $H$ \& E, original magnification $\times$ 150. Reprinted with permission from Rochkind et al.: Laser Surg Med 7:441-443, 1987.

The possible mechanism of action of phototherapy on the nervous tissue with respect to peripheral nerve regeneration has been provided by in vitro studies, which show that phototherapy induced massive neurite sprouting and outgrowth in cultured neuronal cells ${ }^{31}$ as well as Schwann cell proliferation. ${ }^{30}$ It has also been suggested that phototherapy may enhance recovery of neurons from injury by altering mitochondrial oxidative metabolism, ${ }^{3}$ and guiding neuronal growth cones in vitro, perhaps due to the interaction with cytoplasmic proteins and, in particular, to the enhancement of actin polymerization at the leading axon edge. ${ }^{4} \mathrm{~A}$ possible molecular explanation was provided by demonstrating an increase in growth-associated protein-43 immunoreactivity in the early stages of rat sciatic nerve regeneration after phototherapy.25 Another study ${ }^{26}$ showed that application of phototherapy upregulates calcitonin gene-related peptide mRNA expression in facial motor nuclei after axotomy. By altering the intensity or temporal pattern of injury-induced calcitonin gene-related peptide expression, phototherapy may thus optimize the rate of regeneration and target innervation and neuronal survival of axotomized neurons. The purpose of this review is to accumulate data pertaining to the effectiveness of 780-nm laser phototherapy in treating a severely injured peripheral nerve, based on our long-term research.

\section{Animal Studies}

\section{Experimental Peripheral Nerve Crush Injury}

Laser phototherapy significantly improves recovery of the injured peripheral nerve, $, 1,14,20,24$ and in addition, decreases posttraumatic retrograde degeneration of the neurons in the corresponding segments of the spinal cord..$^{15}$ Our previous studies investigating the effects of low-power laser irradiation on an injured peripheral nerve in rats have found that it provides: 1) immediate protective effects that increase the functional activity of the injured peripheral nerve; ${ }^{21}$ 2) maintenance of functional activity of the injured nerve over time, ${ }^{14} 3$ ) a decrease or prevention of scar tissue formation at the site of injury (Fig. 1); ${ }^{20}$ 4) prevention or decreased degeneration in corresponding motor neurons of the spinal cord (Fig. 2) ${ }^{15}$ and 5) an increase in the rate of axonal growth and myelinization (Fig. 3). ${ }^{14,24}$

Moreover, direct laser irradiation of the spinal cord
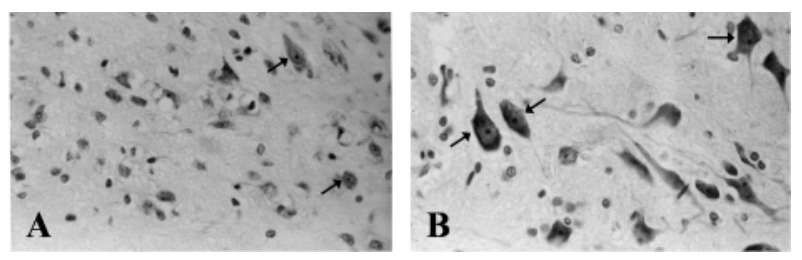

FIG. 2. Paraffin sections from the anterior horn of corresponding segments of the rat spinal cord 14 days after a crush injury to the sciatic nerve, showing the spinal cord response to laser treatment of the injured peripheral nerve. Damaged as well as intact neurons were found in both laser-treated and control animals. a: Section from a control animal shows extensive chromatolysis and cytoplasmic atrophy found in $40 \%$ of the motor neurons (arrows). b: Section from a laser-treated animal shows minimal degenerative changes found in $20 \%$ of the motor neurons (arrows). Cresyl fast violet, original magnification $\times 800$. Reprinted with permission from Rochkind et al.: Spine 15: 6-10, 1990.

improves recovery of the corresponding injured peripheral nerve. ${ }^{19,24}$ These results suggest that laser phototherapy accelerates and improves the regeneration of the injured peripheral nerve.

\section{Regeneration of the Transected Sciatic Nerve in the Rat After Primary Anastomosis}

In acute cases in which a peripheral nerve is completely transected, the treatment of choice is direct anastomosis. The means of enhancing regeneration are essential, because degeneration is always inevitable in severely damaged peripheral nerves.

A double-blind randomized study ${ }^{24}$ evaluated the therapeutic effect of 780-nm laser phototherapy on peripheral nerve regeneration after complete transection and direct anastomosis of the rat sciatic nerve. After this procedure, 13 of 24 rats received postoperative transcutaneous laser treatment, for 30 minutes daily on 21 consecutive days, to corresponding segments of the spinal cord and to the injured sciatic nerve. Positive somatosensory evoked responses were found in $69.2 \%$ of the laser-treated rats $(\mathrm{p}=0.019)$, compared with $18.2 \%$ of the untreated rats. Immunohistochemical staining in the laser-treated group showed a significantly increased total number of
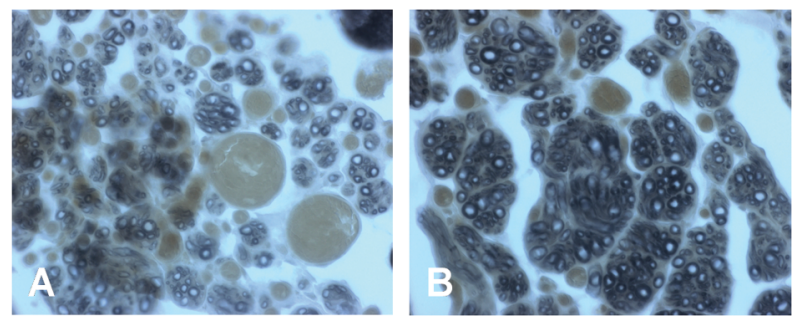

FIG. 3. Photomicrographs of semithin sections stained with toluidine blue showing the axonal response to laser treatment of the injured (crushed) peripheral nerve in the rat. One group of rats was treated using laser phototherapy for 20 consecutive days after injury. Twentyone days after injury the nerves were excised and stained. a: Site of crush injury in an untreated nerve showing nerve fibers that appear to be smaller and mostly nonmyelinated. Numerous macrophages and phagocytes are observed. b: Site of crush injury in a laser-treated nerve demonstrating that most axons are ensheathed with myelin and very few infiltrating macrophages are observed. 

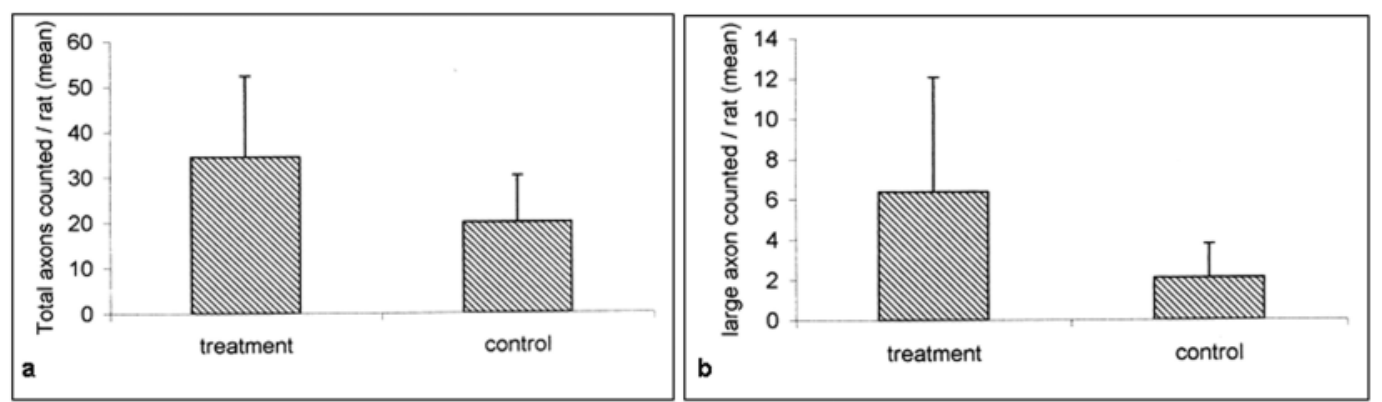

FIG. 4. Bar graphs illustrating the results of a double-blind randomized study evaluating regeneration of the transected rat sciatic nerve after suturing and postoperative low-power laser treatment. a: Graph showing a statistically significant increase in the total number of axons in the laser-treated group $(p=0.026)$, compared with the nontreated control group. b: Graph showing a statistically significant increase in large diameter axons in the laser-treated group $(p=0.021)$ compared with the nontreated control group. Reprinted with permission from Shamir et al: J Reconstruct Microsurg 17:133-138, 2001.

axons $(\mathrm{p}=0.026)$ and better quality of the regeneration process due to more large diameter axons $(p=0.021)$ compared with the nonlaser-treated group (Fig. 4). This study suggests that postoperative laser phototherapy enhances the regenerative processes of peripheral nerves after complete transection and anastomosis.

\section{Regeneration of the Sciatic Nerve in the Rat After Neuro- tube Reconstruction}

In cases in which the peripheral nerve is injured and complete segmental loss exists, the treatment of choice is nerve reconstruction using an autogenous nerve graft. The use of a regenerating guiding tube for the reconstruction of segmental loss of a peripheral nerve has some advantages compared with the regular nerve grafting procedure.

A pilot study was conducted to evaluate the efficacy of 780-nm laser phototherapy on the acceleration of axonal growth and regeneration after experimental peripheral nerve reconstruction using a guiding tube. ${ }^{22}$ The 5 -mm segment of the right sciatic nerve was removed and proximal and distal parts were inserted into a bioabsorbable polyglycolic acid neurotube (Fig. 5). The rats were divided into 2 groups (laser-treated and nonlaser-treated).

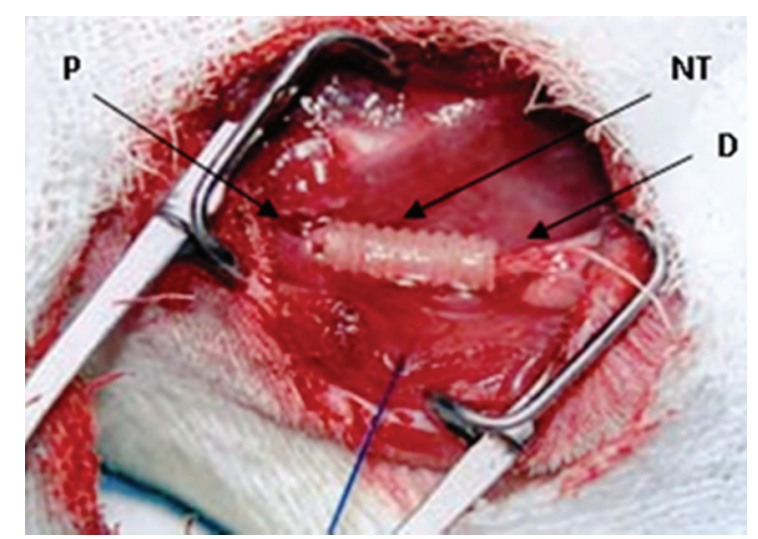

FIG. 5. Intraoperative photograph of the neurotube (NT) reconstruction procedure. A neurotube is placed between the proximal $(P)$ and distal (D) parts of the rat sciatic nerve for the reconnection of a $0.5 \mathrm{~cm}$ nerve defect (arrows). Reprinted with permission from Rochkind et al.: Photomed Laser Surg 25: 137-143, 2007.
Postoperative low-power laser irradiation was applied for 30 minutes transcutaneously on the transplanted peripheral nerve area and corresponding segments of the spinal cord on 14 consecutive days. Conductivity of the sciatic nerve was determined by stimulating the sciatic nerve and recording the somatosensory evoked potentials from the scalp. Three months after surgery, somatosensory evoked potentials were found in $70 \%$ of the rats in the laser-treated group in comparison with $40 \%$ of the rats in the nonlaser-treated group. Morphologically, the previously transected nerve had good reconnection in both groups and the neurotube had dissolved (Fig. 6). The growth of myelinated axons, which crossed through the composite neurotube, was found and the continuation of axonal sprouting through the area of the tube to the distal part of the nerve was recognized. The laser-treated group showed more intensive axonal growth compared with the control group.

\section{Influence of Laser Irradiation on Nerve Cell Growth In Vitro as a Potential Procedure for Neuronal Injury}

Neuronal loss and degeneration resulting from peripheral nerve injuries has lead us to explore the possibility of using laser phototherapy on cells as a method of preventing or decreasing this phenomena. In this work, ${ }^{17}$ the effect of 780 -nm laser phototherapy was investigated on axonal sprouting and cell size of embryonic

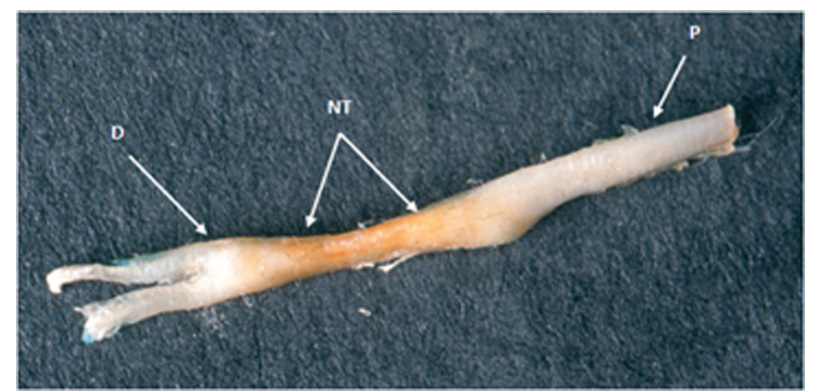

FIG. 6. Photograph of the sciatic nerve of an adult rat 3 months after neurotube (NT) reconstruction. The neurotube recreated the anatomical connection of the previously transected and divided nerve, and a distance of $0.5 \mathrm{~cm}$ was recreated. Reprinted with permission from Rochkind et al.: Photomed Laser Surg 25: 137-143, 2007. 
S. Rochkind
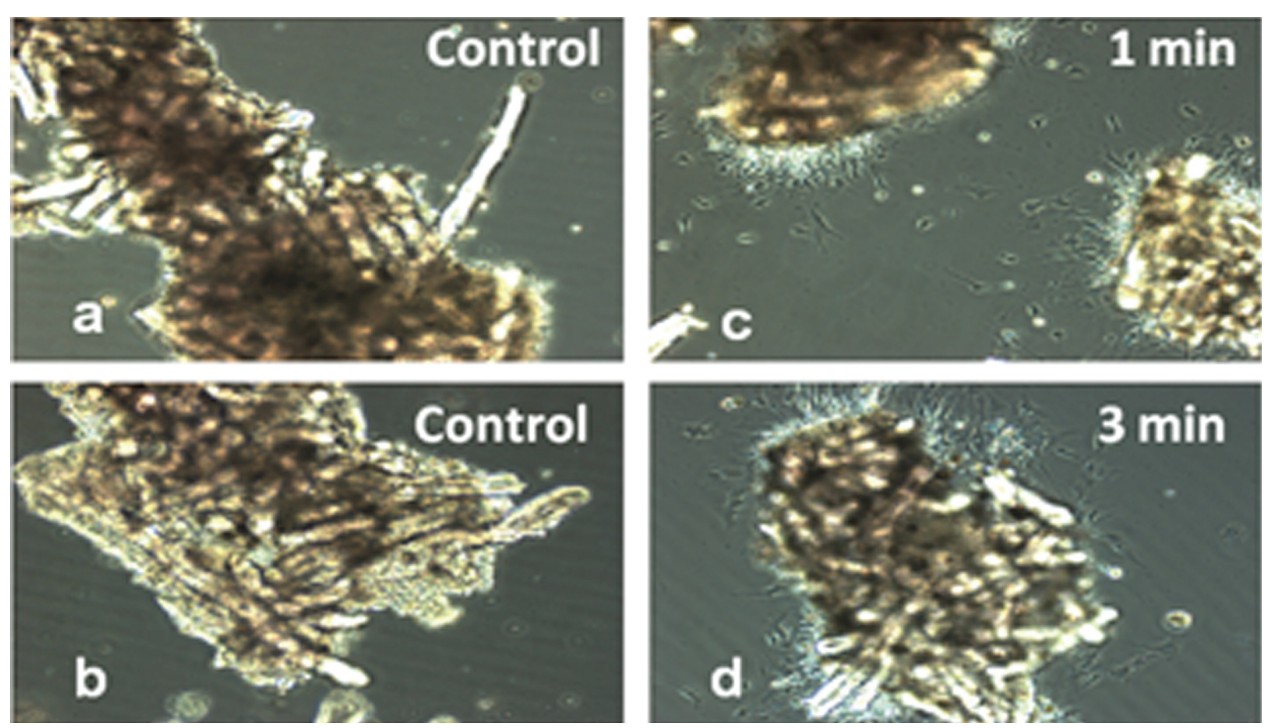

FIG. 7. Photographs showing the effect of 780-nm low-power laser phototherapy on the initial sprouting and migration of embryonic rat brain cells from microcarriers (DE-53) in NeuroGel. Only 1 day after the transfer to stationary cultures in NeuroGel, initial sprouting and cellular migration was observed in irradiated cultures but not in controls. a and b: Nonirradiated controls. c: Cells after a single irradiation dose of $50 \mathrm{~mW}$ for $1 \mathrm{~min}$. d: Cells after a single irradiation dose of $50 \mathrm{~mW}$ for 3 min. Original magnification $\times 200$. Reprinted with permission from Rochkind et al.: Lasers Surg Med, in press, 2009.

rat brain cells on microcarriers embedded in NeuroGel ${ }^{23}$ in culture. Whole brains were dissected from 16-day-old Sprague-Dawley rat embryos. After mechanical dissociation, cells were seeded directly in NeuroGel or suspended in positively charged cylindrical microcarriers. Singlecell microcarrier aggregates were either irradiated with the laser within 1 hour after seeding, or cultured without irradiation. Neurogel (hyaluronic acid and laminin) was enriched with growth factors (brain-derived neurotrophic factor and insulin-like growth factor-I).$^{23}$

To optimize energy density for activation of nerve cell culture, 10, 30, 50,110, 160, 200, and $250 \mathrm{~mW}$ laser powers were applied. Dissociated cells or cell microcarrier aggregates embedded in NeuroGel were irradiated for $1,3,4$, or 7 minutes. Cell cultures were fixed with $4 \%$ paraformaldehyde and incubated with antibodies against neural cell markers (mouse anti-rat microtubule-associ-
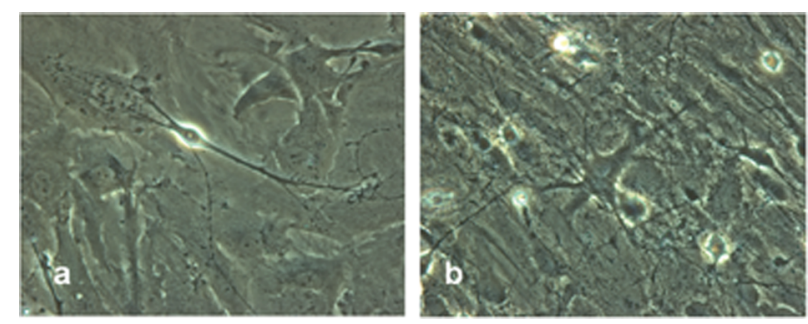

FIG. 8. Photographs of the effect of 780-nm laser treatment on perikarya and fibers of nerve cells derived from rat embryonic brain. Dissociated brain cells were embedded in NeuroGel and were either exposed to a single radiation dose of $50 \mathrm{~mW}$ for $3 \mathrm{~min}(\mathrm{~b})$, or were nonirradiated controls (a). Large neural cells exhibiting thick fibers were observed after 8 days in in vitro irradiated cultures (b). Original magnification $\times 200$. Reprinted with permission from Rochkind et al.: Lasers Surg Med, in press, 2009 ated protein). Cells were then washed and incubated with Texas Red conjugated goat anti-mouse immunoglobulin.

A rapid sprouting of nerve processes from the irradiated cell microcarrier aggregates was detected within 24 hours after seeding (Fig. 7). The extension of nerve fibers was followed by active neuronal migration. Differences between controls and irradiated stationary dissociated brain cultures became evident at approximately the end of the 1st week of cultivation; several neurons in the irradiated cultures exhibited large perikarya and thick elongated processes (Fig. 8). Furthermore, during the next 2-3 weeks of cultivation, neurons in the irradiated cultures developed a dense, branched, interconnected network of neuronal fibers (Fig. 9).

\section{Clinical Study}

Based on the outcome of these animal studies, a clinical double-blind, placebo-controlled, randomized study

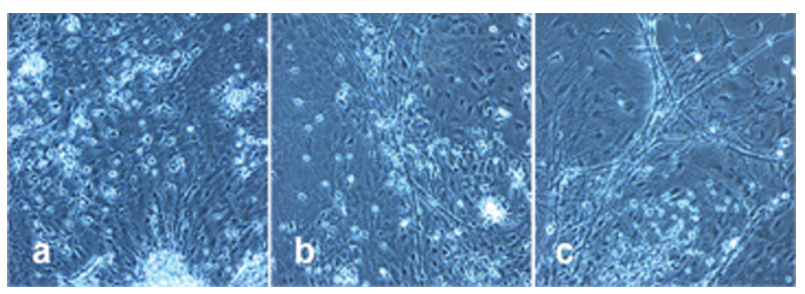

FIG. 9. Photographs of the effect of $780-\mathrm{nm}$ laser treatment on sprouting of rat embryonic brain neurons. Floating cell microcarrier aggregates were transferred to stationary cultures and exposed to a single radiation dose of $50 \mathrm{~mW}$. a: Cells from an untreated control. b: Cells after 4 minutes of radiation. c: Cells after 7 minutes of radiation. Note the branching of huge processes in the laser-treated cultures ( $b$ and $c$ ) after 7 days in NeuroGel. Original magnification $\times 100$. Reprinted with permission from Rochkind et al.: Lasers Surg Med, in press, 2009 


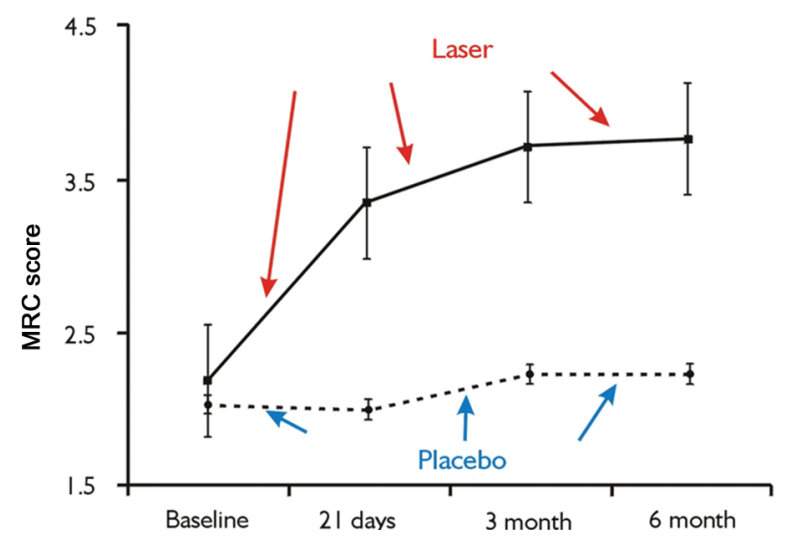

FiG. 10. Graph of the motor function follow-up in injured patients who underwent either 780-nm laser phototherapy or placebo treatment. Mean motor function $( \pm \mathrm{SD}$ ) of all affected muscles was examined in injured patients using the Medical Research Council (MRC) Grading System. The analysis of the results showed that at baseline the 780-nm laser-treated and placebo groups were in clinically similar conditions ( $p$ $=0.887$ ). The analysis of motor function during the 6-month follow-up period compared with baseline showed statistically significant improvement $(p=0.0001)$ in the laser-treated group compared with the placebo group. Reprinted with permission from Rochkind et al.: Photomed Laser Surg 25: 436-442, 2007.

was performed to measure the effectiveness of $780-\mathrm{nm}$ low-power laser phototherapy on patients who had been suffering from incomplete peripheral nerve and brachial plexus injuries for 6 months to several years. ${ }^{16}$ Most of these patients were discharged by orthopedists, neurosurgeons, or plastic surgeons without further treatment. In this study, 18 patients with a history of traumatic peripheral nerve/brachial plexus injury with a stable neurological deficit and a significant weakness were randomly divided to receive either 780-nm laser or placebo (nonactive light) radiation. The mean duration from injury to treatment was 7 months in the laser-treated group and 11.5 months in the placebo group.

The laser or placebo treatment was applied transcutaneously for 5 hours each day for 21 consecutive days ( 3 hours to the injured area of the peripheral nerve and 2 hours to the corresponding segments of the spinal cord). The laser or placebo device was placed approximately 40 $\mathrm{cm}$ from the skin treatment point, focused on the injured area of the peripheral nerve or corresponding level of the spine (area of the corresponding segments of the spinal cord).

Laser irradiation was performed transcutaneously directly above the projection of the corresponding segments of the spinal cord, which was divided into 2 intravertebral levels. Each level was irradiated for 60 minutes a day $(150$ $\left.\mathrm{J} / \mathrm{mm}^{2}\right)$, for a total of 120 minutes each day $\left(300 \mathrm{~J} / \mathrm{mm}^{2}\right)$. Laser irradiation was performed transcutaneously directly above the projection of the injured nerve, which was divided into 3 parts: the proximal, injured, and distal parts of the nerve. Each section was irradiated for 60 minutes a day $\left(150 \mathrm{~J} / \mathrm{mm}^{2}\right)$, for a total of 180 minutes each day $(450$ $\left.\mathrm{J} / \mathrm{mm}^{2}\right)$. The irradiated spot size was $3 \times 2 \mathrm{~mm}\left(6 \mathrm{~mm}^{2}\right)$. The penetration of the near-infrared $780 \mathrm{~nm}$ wavelength is approximately $4 \mathrm{~cm}$.

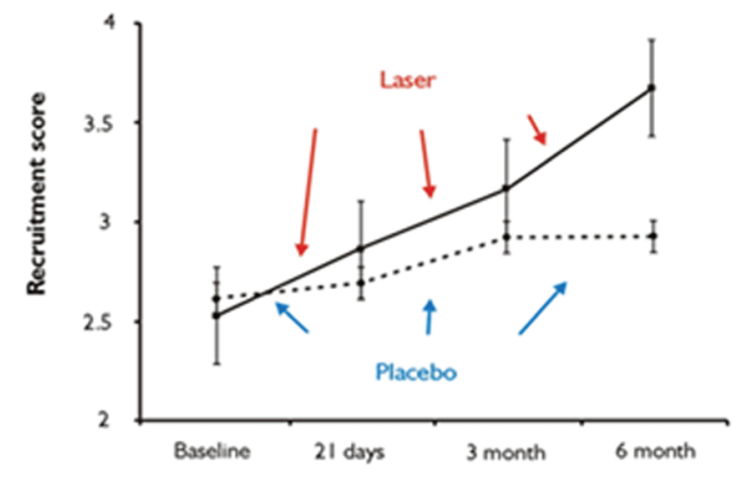

FIG. 11. Graph of the motor unit recruitment in injured patients who underwent either 780-nm laser phototherapy or placebo treatment. Motor unit recruitment, the mean of all examined muscles ( \pm SD), was monitored in injured patients. The 780-nm laser-treated and placebo groups were in similar conditions at baseline $(p=0.934)$. In the laser-treated group, statistically significant improvement $(p=0.0006)$ was found in motor unit recruitment during the 6-month follow-up period, compared with the placebo group. Reprinted with permission from Rochkind et al.: Photomed Laser Surg 25: 436-442, 2007.

The analysis of the results of this trial in the lasertreated group showed statistically significant improvement in motor function in the previously partially paralyzed limbs compared with the placebo group, whereas no statistical significance in neurological status was found (Fig. 10). Mean motor function of the most influential (functionally dominant) muscle for movement of the affected limb showed a statistically significant improvement in the laser-treated group compared with the placebo group. The function was improved mostly by increasing the power of the dominant muscles and not the intrinsic muscles.

Electrophysiological observation during the study supplied us with important diagnostic information and helped to determine the degree of functional recovery in nerve-injured patients. The electrophysiological analysis also showed statistically significant improvement in recruitment of voluntary muscle activity in the laser-treated group compared with the placebo group (Fig. 11).

This study is not the final judgement regarding 780-nm laser phototherapy in peripheral nerve-injured patients. The disadvantages of this study are the small number of patients, the different nerves treated, and the different causes of injury. Nevertheless, this pilot study suggests that in peripheral nerve-injured patients, 780-nm low-power laser phototherapy can progressively improve peripheral nerve function, which has led us to continue this study in a prospective multicenter trial.

\section{Conclusions}

An extensive review article published in $2005^{6}$ revealed that most experimental studies showed that phototherapy promoted the recovery of a severely injured peripheral nerve. This review makes it possible to suggest that the time has arrived for broader clinical trials to be conducted. The significance of the experimental and clinical studies is the introduction of new laser technology for treatment of severe nerve injury. 


\section{Disclaimer}

The authors report no conflict of interest concerning the materials or methods used in this study or the findings specified in this paper.

\section{References}

1. Anders JJ, Borke RC, Woolery SK, Van de Merwe WP: Low power laser irradiation alters the rate of regeneration of the rat facial nerve. Lasers Surg Med 13:72-82, 1993

2. Belzberg AJ, Dorsi MJ, Storm PB, Moriarity JL: Surgical repair of brachial plexus injury: a multinational survey of experienced peripheral nerve surgeons. J Neurosurg 101:365376, 2004

3. Eells JT, Henry MM, Summerfelt P, Wong-Riley MT, Buchmann EV, Kane M, et al: Therapeutic photobiomodulation for methanol-induced retinal toxicity. Proc Natl Acad Sci U S A 100:3439-3444, 2003

4. Ehrlicher A, Betz T, Stuhrmann B, Koch D, Milner V, Raizen MG, et al: Guiding neuronal growth with light. Proc Natl Acad Sci U S A 99:16024-16028, 2002

5. Gigo-Benato D, Geuna S, de Castro Rodrigues A, Tos P, Fornaro M, Boux E, et al: Low-power laser biostimulation enhances nerve repair after end-to-side neurorrhaphy: a double-blind randomized study in the rat median nerve model. Lasers Med Sci 19:57-65, 2004

6. Gigo-Benato D, Geuna S, Rochkind S: Phototherapy for enhancing peripheral nerve repair: a review of the literature. Muscle Nerve 31:694-701, 2005

7. Kline DG, Hackett ER: Reappraisal of timing for exploration of civilian peripheral nerve injuries. Surgery 78:54-65, 1975

8. MacKinnon SE, Dellon AL: Surgery of the Peripheral Nerve. New York: Thieme Medical Publishers, Inc., 1988

9. Midha R: Mechanism and pathology of injury, in Kim DH, Midha R, Murovic JA, Spinner RJ, Tiel R (eds): Kline \& Hudson's Nerve Injuries, ed 2. St. Louis: Saunders Elsevier, 2008, pp 23-42

10. Narakas A: Surgical treatment of traction injuries of the brachial plexus. Clin Orthop Relat Res 133:71-90, 1978

11. Noble J, Munro CA, Prasad VS, Midha R: Analysis of upper and lower extremity peripheral nerve injuries in a population of patients with multiple injuries. J Trauma 45:116-122, 1998

12. Rochkind S: Stimulation effect of laser energy on the regeneration of traumatically injured peripheral nerves. Morphogenesis Regeneration 83:25-27, 1978

13. Rochkind S, Alon M: Microsurgical management of old injuries of the peripheral nerve and brachial plexus. J Reconstr Microsurg 16:541-546, 2000

14. Rochkind S, Barr-Nea L, Razon N, Bartal A, Schwartz M: Stimulatory effect of HeNe Laser low-dose laser on injured sciatic nerves of rats. Neurosurgery 20:843-847, 1987

15. Rochkind S, Barr-Nea L, Volger I: Spinal cord response to laser treatment of injured peripheral nerve. Spine 15:6-10, 1990

16. Rochkind S, Drory V, Alon M, Nissan M, Ouaknine GE: The treatment of incomplete peripheral nerve injuries using a new modality-laser phototherapy $(780 \mathrm{~nm})$. Photomed Laser Surg 25:436-442, 2007

17. Rochkind S, El-Ani D, Hayun T, Nevo Z, Shahar A: Increase of neuronal sprouting and migration using $780 \mathrm{~nm}$ laser phototherapy as procedure for cell therapy. Laser Surg Med 2009

18. Rochkind S, Filmar G, Kluger Y, Alon M: Microsurgery of penetrating peripheral nerve injuries: pre, intra- and postoperative analysis and results. Acta Neurochir Suppl 100:21-24, 2007

19. Rochkind S, Nissan M, Alon M, Shamir M: Salame: Effects of laser irradiation on the spinal cord for the regeneration of crushed peripheral nerves in rats. Lasers Surg Med 28:216219, 2001

20. Rochkind S, Nissan M, Barr-Nea L, Schwartz M, Bartal A: Response of peripheral nerve to HeNe Laser: experimental studies. Lasers Surg Med 7:441-443, 1987

21. Rochkind S, Nissan M: Lubart, Avram J, Bartal A: The in vivo nerve response to direct low-energy laser irradiation. Acta Neurochir (Wien) 94:74-77, 1988

22. Rochkind S, Leider-Trejo L, Nissan M, Shamir M, Kharenko O, Alon M: Efficacy of 780-nm laser phototherapy on peripheral nerve regeneration after neurotube reconstruction procedure (double-blind randomized study). Photomed Laser Surg 25:137-143, 2007

23. Rochkind S, Shahar A, Fliss D, El-Ani D, Astachov L, Hayon $\mathrm{T}$, et al: Development of a tissue-engineered composite implant for treating traumatic paraplegia in rats. Eur Spine $\mathbf{J}$ 15:234-245, 2006

24. Shamir MH, Rochkind S, Sandbank J, Alon M: Double-blind randomized study evaluating regeneration of the rat transected sciatic nerve after suturing and postoperative low power laser treatment. J Reconstr Microsurg 17:133-138, 2001

25. Shin DH, Lee E, Hyun JK, Lee SJ, Chang YP, Kim JW, et al: Growth-associated protein-43 is elevated in the injured rat sciatic nerve after low power laser irradiation. Neurosci Lett 344:71-74, 2003

26. Snyder SK, Byrnes KR, Borke RC, Sanchez A, Anders JJ: Quantification of calcitonin gene-related peptide mRNA and neuronal cell death in facial motor nuclei following axotomy and $633 \mathrm{~nm}$ low power laser treatment. Lasers Surg Med 31:216-222, 2002

27. Spinner RJ: Operative care and techniques, in Kim DH, Midha R, Murovic JA, Spinner RJ, Tiel R (eds): Kline \& Hudson's Nerve Injuries, ed 2. St Louis: Saunders Elsevier 2008, pp 87-105

28. Sunderland S: Nerves and Nerve Injuries, ed 2. Edinburgh: Churchill Livingstone, 1978

29. Terzis JK, Smith KL: The Peripheral Nerve: Structure, Function and Reconstruction. New York: Raven Press, 1990

30. Van Breugel HH, Bar PR: HeNe laser irradiation affects proliferation of cultured rat schwann cells in a dose-dependent manner. J Neurocytol 22:185-190, 1993

31. Wollman Y, Rochkind S, Simantov R: Low power laser irradiation enhances migration and neurite sprouting of cultured rat embryonal brain cells. Neurol Res 18:467-470, 1996

Manuscript submitted October 11, 2008.

Accepted November 19, 2008

Address correspondence to: Shimon Rochkind, M.D., Division of Peripheral Nerve Reconstruction, Department of Neurosurgery, Tel Aviv Sourasky Medical Center, 6 Weizmann Street, Tel Aviv, Israel 64239.email: rochkind@zahav.net.il. 